\title{
Virtual Bronchoscopic Navigation Improves the Diagnostic Yield of Radial-Endobronchial Ultrasound for Peripheral Pulmonary Lesions with Involved Bronchi on CT
}

\author{
Fumihiro Asano ${ }^{1}$, Naofumi Shinagawa ${ }^{2}$, Takashi Ishida ${ }^{3}$, Akifumi Tsuzuku ${ }^{1}$, \\ Motoko Tachihara ${ }^{3}$, Kenya Kanazawa ${ }^{3}$, Noriyuki Yamada ${ }^{2}$, \\ Satoshi Oizumi ${ }^{2}$ and Hiroshi Moriya ${ }^{4}$
}

\begin{abstract}
Objective Bronchoscopy using radial-endobronchial ultrasound (R-EBUS) and virtual bronchoscopic navigation (VBN) is a promising method for diagnosing peripheral pulmonary lesions. We previously performed a randomized comparative trial (RCT) (i.e., VBN combined with EBUS RCT) involving patients with 30-mm or smaller peripheral pulmonary lesions and found that the addition of VBN to R-EBUS improved the diagnostic yield. In the present study, we performed a retrospective subanalysis in order to identify patients for whom VBN is useful.

Methods The per-protocol population (194 cases) of the VBN combined with EBUS RCT was divided into subgroups based on the lesion size, lung lobe containing the lesion, lesion location, presence or absence of involved bronchi (bronchus sign) on thin-section $\mathrm{CT}$ and whether the lesion was detected on posterioranterior (P-A) radiographs. The difference in the diagnostic yield between the VBN-assisted (VBNA) and non-VBN-assisted (NVBNA) groups was investigated.

Results Within the bronchus sign-positive subgroup, the diagnostic yield in the VBNA and NVBNA groups was $94.4 \%$ (68/72) and $77.8 \%$ (56/72), respectively, showing a significantly higher yield in the VBNA group ( $\mathrm{p}=0.004$; odds ratio: 4.9). The yield was particularly high for lesions smaller than $20 \mathrm{~mm}(94.6 \%$ vs. $70.7 \%$; $\mathrm{p}=0.006)$, lesions located in the peripheral third of the lung field $(95.1 \%$ vs. $71.4 \%$; $=0.005)$ and lesions invisible on P-A radiographs $(90.0 \%$ vs. $41.7 \%$; $\mathrm{p}=0.026)$.

Conclusion VBN improves the diagnostic yield when combined with R-EBUS to assess lesions exhibiting involved bronchi on CT images.
\end{abstract}

Key words: bronchoscopy, lung cancer, solitary pulmonary nodule, transbronchial biopsy, virtual bronchoscopy

(Intern Med 54: 1021-1025, 2015)

(DOI: 10.2169/internalmedicine.54.3497)

\section{Introduction}

The widespread use of computed tomography (CT) has increased the detection rate of peripheral pulmonary lesions. However, many of these lesions are benign and thus detect- ing early lung cancer in patients with such lesions is crucial (1). Peripheral pulmonary lesions are definitively diagnosed using surgical biopsies, transthoracic needle aspiration (TTNA) and/or transbronchial biopsies (2). Bronchoscopy in particular has the advantage of having less complications, although its diagnostic yield is insufficient (3). The American

\footnotetext{
${ }^{1}$ Department of Pulmonary Medicine, Gifu Prefectural General Medical Center, Japan, ${ }^{2}$ The First Department of Medicine, Hokkaido University School of Medicine, Japan, ${ }^{3}$ Department of Pulmonary Medicine, Fukushima Medical University, Japan and ${ }^{4}$ Diagnostic Imaging Center, Ohara General Hospital, Japan

Received for publication June 16, 2014; Accepted for publication September 15, 2014

Correspondence to Dr. Fumihiro Asano, asano-fm@ceres.ocn.ne.jp
} 
College of Chest Physicians (ACCP) guidelines recommend the use of radial endobronchial ultrasound (R-EBUS) for the bronchoscopic diagnosis of peripheral pulmonary lesions when appropriate devices and a skilled operator are available (3). R-EBUS identifies lesions and sampling sites in real time using a radial-type ultrasound probe. Employing this method, biopsies may be applied repeatedly at the same site with the concomitant use of a guide sheath (GS) (4). In a previous meta-analysis, the pooled sensitivity and specificity of R-EBUS for the detection of lung cancer in peripheral pulmonary lesions were $73 \%$ [95\% confidence interval (CI): 70-76\% ] and 100\% (95\% CI: 99-100\%), respectively (5). However, some lesions cannot be reached with the radial probe because the EBUS system does not include a navigation device $(6,7)$.

Virtual bronchoscopic navigation (VBN) is a method in which virtual bronchoscopy (VB) images of the bronchial path to the peripheral lesion are produced and used as a guide to navigate the bronchoscope $(8,9)$. In order to confirm the arrival of the bronchoscope at the lesion site, it is necessary to combine VBN with another procedure. For instance, the combination of VBN and EBUS-GS shows a high diagnostic yield of $63.3-84.4 \%$ for peripheral pulmonary lesions and $44.4-75.9 \%$ for $20-\mathrm{mm}$ or smaller lesions (10-14). We also previously demonstrated in a multicenter randomized comparative trial (RCT) that the addition of VBN to EBUS-GS significantly improves the diagnostic yield and shortens the examination time (VBN combined with EBUS RCT) (13). Briefly, patients with 30-mm or smaller peripheral pulmonary lesions suspected to be lung cancer were randomly divided into two groups. In the VBNassisted (VBNA) group, a thin bronchoscope was guided to the lesion using a VBN system, by referring to the axial CT images in the non-VBN-assisted (NVBNA) group, followed by a biopsy. The diagnostic yield in the VBNA group according to the per-protocol analysis was $80.8 \%$, being significantly higher than that $(67.4 \%)$ observed in the NVBNA group $(\mathrm{p}=0.032)$. Meanwhile, the diagnostic yield was $75.9 \%$ vs. $59.3 \%$ for $<20-\mathrm{mm}$ lesions $(\mathrm{p}=0.056)$ and $87.8 \%$ vs. $80.6 \%$ for $20-30-\mathrm{mm}$ lesions $(\mathrm{p}=0.382)$ in the VBNA and NVBNA groups, respectively.

Since VBN requires the use of thin-section CT under conditions recommended for the system, problems involving additional costs and increased exposure arise in cases requiring repeat CT images. Accordingly, the application of VBN in medical practice necessitates patient selection. In the present study, we performed a subanalysis of an RCT of VBN combined with EBUS (13) in order to identify patients for whom the addition of VBN to R-EBUS is useful.

\section{Materials and Methods}

Data from the per-protocol population (194 cases) of the VBN combined with EBUS RCT (13) that included all randomized patients undergoing planned bronchoscopic procedures for peripheral lesions were statistically analyzed. Two pulmonologists who were not informed of the diagnostic results individually evaluated the lesion size, lung lobe containing the lesion, lesion location and presence or absence of involved bronchi (bronchus sign) (15) on thin-section CT images (slice thickness: $0.5-1 \mathrm{~mm}$ ) used for bronchoscopy and determined whether the lesion was detected on posterior-anterior ( $\mathrm{P}-\mathrm{A})$ radiographs. The location of the lesion was classified into central, intermediate and peripheral thirds according to the distance from the hilum based on the study of Baaklini and coworkers (16). When the findings of the two pulmonologists were inconsistent, a third pulmonologist made the final judgment. The difference in the diagnostic yield between the VBNA and NVBNA groups was investigated for each item. The institutional ethics committee approved the study.

\section{Statistical analysis}

Dichotomous variables were analyzed using the Pearson $\chi 2$ test or Fisher's exact test. All p-values were two-sided. A $\mathrm{p}$ value of $<0.05$ was considered to indicate a statistically significant difference. The Bonferroni correction was not used to address the multiplicity problem in this study. All data were statistically analyzed using the IBM SPSS Statistics software program, version 19 (SPSS, Chicago, USA).

\section{Results}

Regarding the presence or absence of the bronchus sign, within the bronchus sign-positive subgroup, the diagnostic yield in the VBNA and NVBNA groups was $94.4 \%$ and $77.8 \%$, respectively, showing a significantly higher yield in the VBNA group [ $\mathrm{p}=0.004$; odds ratio: $4.9 ; 95 \%$ confidence interval (CI): 1.5-15.4]. In the bronchus sign-negative subgroup, the diagnostic yield was not significantly different between the two groups. In addition, there were no significant differences in the diagnostic yield between the VBNA and NVBNA groups in any of the subgroups in terms of the lung lobe containing the lesion, lesion location and whether the lesion was detected on P-A radiographs (Table 1).

Within the bronchus sign-positive subgroup, the diagnostic yield was significantly higher in the VBNA group among the patients with a lesion size of $<20 \mathrm{~mm}(94.6 \%$ vs. 70.7\%; $\mathrm{p}=0.006$; odds ratio: 7.2; 95\% CI: 1.5-35.0), lesions located in the peripheral third of the lung field (95.1\% vs. 71.4\%; $\mathrm{p}=0.005$; odds ratio: 7.8 ; 95\% CI: 1.6-38.6) and invisible lesions on $\mathrm{P}-\mathrm{A}$ radiographs $(90.0 \%$ vs. $41.7 \%$; $\mathrm{p}=$ 0.026; odds ratio: 12.6 ; $95 \% \mathrm{CI}: 1.2-133.9)$. There were no significant differences in the diagnostic yield between the two groups for any other items (Table 2).

\section{Discussion}

We determined whether VBN improves the diagnostic yield of R-EBUS for peripheral pulmonary lesions with involved bronchi. As EBUS is recommended according to the 2013 ACCP guidelines, an increase in the number of cases 
Table 1. Diagnostic Yield According to Each Parameter

\begin{tabular}{|c|c|c|c|c|c|c|}
\hline & & VBNA & NVBNA & $\mathrm{p}$ value & $\begin{array}{l}\text { Odds } \\
\text { ratio }\end{array}$ & $\begin{array}{l}95 \% \\
\text { Confidence } \\
\text { interval }\end{array}$ \\
\hline \multirow[t]{5}{*}{ Lobe } & Rt upper & $27 / 31(87.1)$ & $26 / 34(76.5)$ & 0.270 & 2.1 & $0.6-7.7$ \\
\hline & Rt middle & $12 / 12(100.0)$ & $5 / 6(83.3)$ & 0.146 & 0.8 & $0.6-1.2$ \\
\hline & Rt lower & $15 / 22(68.2)$ & $10 / 18(55.6)$ & 0.412 & 1.7 & $0.5-6.2$ \\
\hline & Lt upper & $19 / 24(79.2)$ & $14 / 20(70.0)$ & 0.484 & 1.6 & $0.4-6.4$ \\
\hline & Lt lower & $7 / 10(70.0)$ & 9/17 (52.9) & 0.324 & 2.1 & $0.4-10.8$ \\
\hline \multirow[t]{3}{*}{ Location } & Central & $1 / 2(50.0)$ & $6 / 10(60.0)$ & 0.682 & 0.7 & $0.03-14.0$ \\
\hline & Intermediate & $34 / 38$ (89.5) & $26 / 35(74.3)$ & 0.090 & 2.9 & $0.8-10.6$ \\
\hline & Peripheral & $45 / 59(76.3)$ & $32 / 50(64.0)$ & 0.161 & 1.8 & $0.8-4.2$ \\
\hline \multirow[t]{2}{*}{ Bronchus sign } & Positive & $68 / 72(94.4)$ & $56 / 72(77.8)$ & 0.004 & 4.9 & $1.5-15.4$ \\
\hline & Negative & $12 / 27(44.4)$ & $8 / 23(34.8)$ & 0.487 & 1.5 & $0.5-4.7$ \\
\hline \multirow{2}{*}{$\begin{array}{l}\text { Posterior-anterior } \\
\text { radiograph }\end{array}$} & Visible & $69 / 82(84.1)$ & $59 / 79(74.7)$ & 0.137 & 1.8 & $0.8-3.9$ \\
\hline & Invisible & $11 / 17(64.7)$ & $5 / 16(31.3)$ & 0.055 & 4.0 & $0.9-17.2$ \\
\hline Total & & $80 / 99(80.8)$ & $64 / 95(67.4)$ & 0.032 & 2.0 & 1.1-3.9 \\
\hline
\end{tabular}

Definition of abbreviations: Lt.: left, NVBNA: non-virtual bronchoscopic navigation-assisted, Rt.: right, VBNA: virtual bronchoscopic navigation-assisted

Data are shown as numbers of lesions/total lesions (\%)

Table 2. Diagnostic Yield According to Each Parameter (Bronchus Sign-Positive Subgroup)

\begin{tabular}{|c|c|c|c|c|c|c|}
\hline & & VBNA & NVBNA & $\mathrm{p}$ value & $\begin{array}{l}\text { Odds } \\
\text { ratio }\end{array}$ & $\begin{array}{l}95 \% \\
\text { Confidence } \\
\text { interval }\end{array}$ \\
\hline \multirow[t]{2}{*}{ Lesion size } & $<20 \mathrm{~mm}$ & $35 / 37(94.6)$ & $29 / 41(70.7)$ & 0.006 & 7.2 & $1.5-35.0$ \\
\hline & $20-30 \mathrm{~mm}$ & $33 / 35(94.3)$ & $27 / 31(87.1)$ & 0.280 & 2.4 & $0.4-14.4$ \\
\hline \multirow[t]{3}{*}{ Location } & Central & $1 / 2(50.0)$ & $6 / 8(75.0)$ & 0.533 & 0.3 & $0.01-8.2$ \\
\hline & Intermediate & $28 / 29(96.6)$ & $25 / 29(86.2)$ & 0.176 & 4.5 & $0.5-42.8$ \\
\hline & Peripheral & $39 / 41(95.1)$ & $25 / 35(71.4)$ & 0.005 & 7.8 & $1.6-38.6$ \\
\hline \multirow[t]{2}{*}{$\begin{array}{l}\text { Posterior-anterior } \\
\text { radiograph }\end{array}$} & Visible & $59 / 62(95.2)$ & $51 / 60(85.0)$ & 0.060 & 3.5 & $0.9-13.5$ \\
\hline & Invisible & $9 / 10(90.0)$ & $5 / 12(41.7)$ & 0.026 & 12.6 & $1.2-133.9$ \\
\hline Total & & $68 / 72(94.4)$ & $56 / 72(77.8)$ & 0.004 & 4.9 & $1.5-15.4$ \\
\hline
\end{tabular}

in which this procedure is applied is expected. However, the characteristics of patients for whom combination treatment with VBN is useful have not been thoroughly investigated. Hence, to the best of our knowledge, this study is the first subclass analysis to show that VBN improves the diagnostic yield when combined with R-EBUS, particularly for lesions exhibiting involved bronchi. Lesions showing an involved bronchus were detected in 144 cases, comprising more than $70 \%$ of all patients, which may account for the significant improvement in the diagnostic yield observed in the total VBNA group (13). The diagnostic yield of conventional bronchoscopy for lesions showing no involved bronchi is low (3). In such cases, transbronchial needle aspiration is reportedly useful (17); however, this procedure was not performed in the current study. Since VBN guides the bronchoscope to the lesion using virtual images of the bronchial route, the routes for lesions without involved bronchi may not have been accurately prepared, thus preventing VBN from achieving any effect. The VBN system is capable of displaying the site and direction of transbronchial needle puncture. At present, TTNA and surgical biopsies are indicated for lesions involving no bronchus, although it is necessary to investigate the effectiveness of transbronchial needle aspiration (TBNA) using VBN.

In this study, the subanalysis clarified that combination treatment with VBN improves the diagnostic yield for lesions showing involved bronchi on thin-section CT images, particularly lesions smaller than $20 \mathrm{~mm}$ and those located in the peripheral third of the lung field or invisible on P-A radiographs. Since the diagnostic yield in these three subgroups on conventional bronchoscopy is reportedly low $(3,16)$, VBN is deemed useful for assessing these le- 
sions. VBN requires the application of thin-slice CT, and repeat CT examinations may be necessary depending on the patient's condition. By limiting the use of VBN to subgroups for whom the diagnostic yield is improved with VBN according to the subanalysis, overall medical expenses can be minimized.

The pooled sensitivity of TTNA for the diagnosis of peripheral bronchogenic carcinoma is reported to be $90 \%$ (95\% CI, 88-91\%) (3), while Ohno et al. reported that the diagnostic yield of CT-guided TTNA for 20-mm or smaller lesions is $77.5 \%$ (18). TTNA is generally performed in such cases due to its high diagnostic yield. However, the diagnostic yield for lesions showing involved bronchi is significantly improved with VBN, reaching $94.4 \%$ in the current subanalysis. In particular, the diagnostic yields for lesions smaller than $20 \mathrm{~mm}$ and those located in the peripheral third of the lungs and invisible on $\mathrm{P}$-A radiographs were high (94.6\%, 95.1\% and 90.0\%, respectively), being comparable with that obtained using TTNA. Regarding the TTNAinduced complication rate, the incidence of pneumothorax requiring drainage is $6.6 \%$ (95\% CI: $6.0-7.2 \%)$, while that including cases not requiring drainage is $15.0 \%$ (95\% CI: 14.0-16.0\%), showing high incidences in both circumstances (19). Meanwhile, the incidence of hemorrhage is $1.0 \%$ (95\% CI: $0.9-1.2 \%$ ), and $17.8 \%$ of such patients require blood transfusions (19). In contrast, the bronchoscopyinduced complication rate is low. Furthermore, no complications directly caused by VBN have been reported, suggesting that bronchoscopy using VBN combined with EBUS is safer than TTNA. Further investigations using RCTs are needed to determine the positions of the two procedures (TTNA and bronchoscopy using VBN combined with EBUS) for the diagnosis of peripheral pulmonary lesions.

This study is associated with some limitations. As the analysis was retrospective, the evidence level is lower than that of prospective studies. In addition, the possibility of biased findings cannot be excluded, and it is possible that significant differences were not detected owing to the weak statistical power of the subanalysis as a result of the small number of cases. Furthermore, confounding factors for each item may have affected the findings, and the most important item for increasing the diagnostic yield using VBN was unclear. In order to clarify these issues, it is necessary to perform a meta-analysis and RCT of VBN, focusing on the subgroups in whom VBN is suggested to be useful.

In conclusion, VBN improves the diagnostic yield when combined with R-EBUS for lesions showing involved bronchi on CT images. As EBUS is recommended according to the ACCP guidelines, the number of cases in which this technique is applied may increase. VBN should be actively used to assess lesions exhibiting involved bronchi, particularly lesions smaller than $20 \mathrm{~mm}$ and those located in the peripheral third of the lung field and/or invisible on P-A radiographs due to the high diagnostic yield of this modality.

The authors state that they have no Conflict of Interest (COI).

\section{Acknowledgement}

The authors are indebted to Dr. Edward Barroga, Associate Professor and Senior Medical Editor of the Department of International Communications of Tokyo Medical University for reviewing and editing the manuscript.

\section{References}

1. Aberle DR, Adams AM, Berg CD, et al. Reduced lung-cancer mortality with low-dose computed tomographic screening. N Engl J Med 365: 395-409, 2011.

2. Gould MK, Fletcher J, Iannettoni MD, et al. Evaluation of patients with pulmonary nodules: when is it lung cancer?: ACCP evidence-based clinical practice guidelines (2nd edition). Chest 132: 108S-130S, 2007.

3. Rivera MP, Mehta AC, Wahidi MM. Establishing the diagnosis of lung cancer: Diagnosis and management of lung cancer, 3rd ed: American College of Chest Physicians evidence-based clinical practice guidelines. Chest 143: e142S-e165S, 2013.

4. Kurimoto N, Miyazawa T, Okimasa S, et al. Endobronchial ultrasonography using a guide sheath increases the ability to diagnose peripheral pulmonary lesions endoscopically. Chest 126: 959-965, 2004.

5. Steinfort DP, Khor YH, Manser RL, Irving LB. Radial probe endobronchial ultrasound for the diagnosis of peripheral lung cancer: systematic review and meta-analysis. Eur Respir J 37: 902-910, 2011.

6. Herth FJ, Eberhardt R, Becker HD, Ernst A. Endobronchial ultrasound-guided transbronchial lung biopsy in fluoroscopically invisible solitary pulmonary nodules: a prospective trial. Chest 129: 147-150, 2006.

7. Kikuchi E, Yamazaki K, Sukoh N, et al. Endobronchial ultrasonography with guide-sheath for peripheral pulmonary lesions. Eur Respir J 24: 533-537, 2004.

8. Asano F, Matsuno Y, Matsushita T, Seko A. Transbronchial diagnosis of a pulmonary peripheral small lesion using an ultrathin bronchoscope with virtual bronchoscopic navigation. J Bronchol 9: 108-111, 2002.

9. Asano F. Virtual bronchoscopic navigation. Clin Chest Med 31: 75-85, 2010.

10. Asahina H, Yamazaki K, Onodera Y, et al. Transbronchial biopsy using endobronchial ultrasonography with a guide sheath and virtual bronchoscopic navigation. Chest 128: 1761-1765, 2005.

11. Asano F, Matsuno Y, Tsuzuku A, et al. Diagnosis of peripheral pulmonary lesions using a bronchoscope insertion guidance system combined with endobronchial ultrasonography with a guide sheath. Lung Cancer 60: 366-373, 2008.

12. Oshige M, Shirakawa T, Nakamura M, et al. Clinical application of virtual bronchoscopic navigation system for peripheral lung lesions. J Bronchology Interv Pulmonol 18: 196-202, 2011.

13. Ishida $T$, Asano F, Yamazaki $K$, et al. Virtual bronchoscopic navigation combined with endobronchial ultrasound to diagnose small peripheral pulmonary lesions: a randomised trial. Thorax 66: 1072-1077, 2011.

14. Tamiya M, Okamoto N, Sasada S, et al. Diagnostic yield of combined bronchoscopy and endobronchial ultrasonography, under LungPoint guidance for small peripheral pulmonary lesions. Respirology 18: 834-839, 2013.

15. Gaeta M, Pandolfo I, Volta S, et al. Bronchus sign on CT in peripheral carcinoma of the lung: value in predicting results of transbronchial biopsy. AJR Am J Roentgenol 157: 1181-1185, 1991.

16. Baaklini WA, Reinoso MA, Gorin AB, et al. Diagnostic yield of fiberoptic bronchoscopy in evaluating solitary pulmonary nodules. Chest 117: 1049-1054, 2000.

17. Chao TY, Chien MT, Lie CH, et al. Endobronchial ultrasonography-guided transbronchial needle aspiration increases 
the diagnostic yield of peripheral pulmonary lesions: a randomized trial. Chest 136: 229-236, 2009.

18. Ohno Y, Hatabu H, Takenaka D, et al. CT-guided transthoracic needle aspiration biopsy of small $(<$ or $=20 \mathrm{~mm})$ solitary pulmonary nodules. AJR Am J Roentgenol 180: 1665-1669, 2003.
19. Wiener RS, Schwartz LM, Woloshin S, Welch HG. Populationbased risk for complications after transthoracic needle lung biopsy of a pulmonary nodule: an analysis of discharge records. Ann Intern Med 155: 137-144, 2011.

(C) 2015 The Japanese Society of Internal Medicine http://www.naika.or.jp/imonline/index.html 